\author{
Military Technical College \\ Kobry Elkobbah, \\ Cairo, Egypt
}

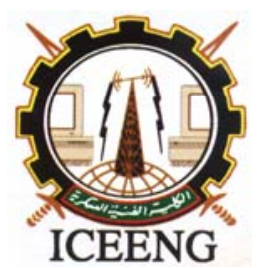

\title{
AUTOMATIC CLASSIFICATION OF MPSK SIGNALS USING STATISTICAL MOMENTS
}

Tarek Helaly ${ }^{*}$

\begin{abstract}
In this paper, an automatic classification algorithm for MPSK signals is proposed. In this algorithm, it is assumed that there is prior information about the received signal to be an MPSK type. The concept of the proposed classification algorithm is based on evaluating the statistical moments of the instantaneous phase of the received signal and using it as a key feature to classify the MPSK signals. The proposed algorithm comprises three main steps: 1) estimation of the instantaneous phase of the received signal, 2) computation of statistical moments of the estimated phase, and 3) decision about the number of phase states of the intercepted signal. The performance of the proposed algorithm is measured in terms of the success rate of classification using computer simulations. It is found that all digital modulation types of interest have been correctly classified with a success rate $>91 \%$ at signal-to-noise ratio, SNR, of $10 \mathrm{~dB}$.
\end{abstract}

\section{Key Words}

Signal Processing, Modulation Recognition, and Automatic Signal Classification.

\section{Introduction}

This paper is concerned with the automatic modulation classification of digital communication signals. Modulation classification is a branch of non-cooperative communication theory such as electronic warfare (EW), surveillance, and threat analysis. Also, modulation classification is extremely important in communication intelligence (COMINT) applications. Modulation classification is neither energy detection nor a normal signal demodulation problem with message extraction. It is something in between. For energy detection only an approximate knowledge of center frequency and signal bandwidth is required, whereas on the other hand for the demodulation with message extraction, the knowledge of the exact values of center frequency, bandwidth, type of used modulation, and all modulation parameters is required.

\footnotetext{
* Associate Lecturer, Electronic Warfare Eng. Department, MTC, Cairo, Egypt.
} 
The signal classifier described in this paper requires values for the signal center frequency that has to be only slightly more precise than those required by an energy detector.

Actually, few articles were published in this area. In [1], a classifier that is based on the artificial neural network is used to classify analogue and digital modulated signals. Several key features are extracted from the received signals and are used in the classification. In [2], a classifier that is based on pattern recognition method is described. The power spectrum is applied as a signal feature that can be used in the classification. The classification algorithm is developed as a software application using digital signal processing technique. In [3], a classifier is proposed for digital modulation schemes based on fourth-order cumulants. In [4], the maximum-likelihood (ML) method to the classification of digital quadrature modulations is used. It is shown that the ML classifier is capable of classifying any finite set of distinctive constellations with zero error rates when the number of available data symbols goes to infinity. In [5], a classifier for MFSK signals contaminated with class-A impulsive noise and transmitted over time varying flat correlated fading channel is developed. In [6], the author introduced a modulation classifier based on the statistical moments of the intercepted signal phase to estimate the number of levels, M, in MPSK signals. The Fourier series expansion of the exact phase distribution of the received signal is used.

In this paper, an automatic classification algorithm for MPSK signals is proposed. The concept of the proposed classification algorithm is based on evaluating the statistical moments of the instantaneous phase of the received signal and using it as a key feature to classify the modulation type of general MPSK signals. It will be explained later that for MPSK signals, the $n^{\text {th }}$ moment (n even) of the phase of the signal is a monotonic increasing function of M. Based on this property, a general hypothesis test is formulated and a decision rule is proposed.

The performance evaluation of the proposed algorithm is measured in terms of the success rate of classification in presence of noise. The proposed algorithm shows a comparable performance with the aforementioned references. But on the other hand it uses simpler formulae for evaluating the $n^{\text {th }}$ order moments and the threshold values.

This paper consists of three main sections. In section 2, the proposed algorithm is explained. In section 3, the performance evaluation of the proposed algorithm is presented. Finally, the conclusions are presented in section 4.

\section{Proposed Algorithm}

The proposed algorithm consists of three main stages; 1) Preprocessing stage which includes the estimation of the instantaneous phase of the received signal, 2) key features extraction stage which includes the computation of statistical moments of the estimated phase, and 3) Classification stage which includes the decision about the number of phase states of the intercepted signal. In the following each stage will be explained in details.

\section{A) Preprocessing stage (Estimation of the instantaneous phase of the received signal)}

In this stage, the instantaneous phase of the received signal is computed. The received signal contains the phase information that can be extracted by means of Inphase-Quadrature (IQ) techniques or by using discrete Hilbert transform [8]. The extracted phase can be represented 


$$
\varphi_{\alpha}(i)=\theta_{\alpha}(i)+v(i) ;-\infty<i<\infty,-\pi \leq \varphi_{\alpha}(i)<\pi
$$

where $\alpha=1,2, \ldots, \log _{2} \mathrm{M}, \mathrm{M}$ is the number of phase levels and $v(i)$ is a random phase attributed to the noise at the classifier input and any other measurement errors. Without loss of generality, $\left\{\varphi_{\alpha}(i)\right\}$ and $\left\{\theta_{\alpha}(i)\right\}$ are independent and identically distributed with zero means.

\section{B) Key feature extraction stage (computation of statistical moments of the estimated phase)}

In this step, statistical moments of the instantaneous phase of the received signal are computed. With equally likely $\mathrm{M}$ phases, the pdf of the instantaneous phase for pure MPSK signal, $\theta_{\alpha}$, can be written as [6],

$$
f_{\theta}(y ; \alpha)=\frac{1}{2^{\alpha}} \sum_{m=1}^{2^{\alpha}} \delta\left(y-\eta_{m}(\alpha)\right)
$$

where $\eta_{m}(\alpha)$ is the phase of the $m^{\text {th }}$ phase states and can be expressed as

$$
\eta_{m}(\alpha)=\frac{\left(2 m-2^{\alpha}-1\right) \pi}{2^{\alpha}} ; m=1,2, \ldots, 2 \alpha, \alpha=1,2, \ldots, \log _{2} \mathrm{M}
$$

In absence of noise, the $n^{\text {th }}$ order moment of $\theta_{\alpha}$ is given by [6]

$$
\begin{aligned}
m_{n}(\alpha) & =\int_{-\pi}^{\pi} y^{n} f_{\theta}(y ; \alpha) d y \\
& =\left\{\begin{array}{cl}
\frac{\pi^{n}}{2^{(n+1) \alpha}} \sum_{i=1}^{2^{\alpha}}\left(2 i-2^{\alpha}-1\right)^{n} & \text { n even }, \alpha \neq 0 \\
0 & \text { n odd or } \alpha=0
\end{array}\right.
\end{aligned}
$$

The even statistical moments of the phase of the received signal for $\alpha=1,2$, and 3 are shown in Fig. 1. This figure shows that the even moments of the phase is a monotonic increasing function with respect to $\alpha$; i.e.

$$
m_{n}(\alpha+1)>m_{n}(\alpha)
$$




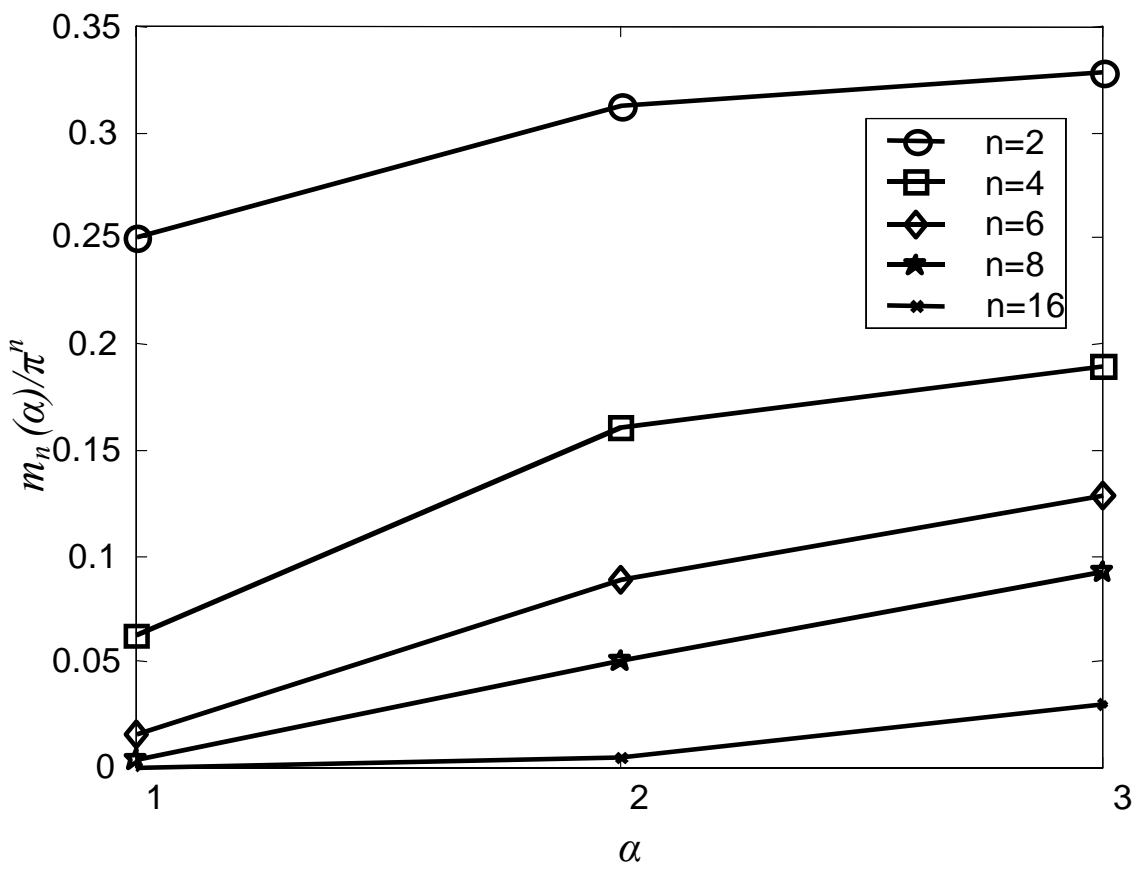

Fig.1. Statistical moments of MPSK signals versus $\alpha$

Thus, it is clear that even moments, $m_{n}(\alpha)$, can be used as a key feature to classify the MPSK signals.

\section{C) Classification stage (Decision about the number of phase states)}

In this step, the decision about the number of phase states of the intercepted signal is achieved. We are interested only in classification of PSK2, PSK4, and PSK8. Extension to higher levels is straight forward to be obtained. The problem of classifying MPSK signals here can be formulated as a hypothesis testing problem based on the phase moments as

$$
\begin{aligned}
& \mathrm{H}_{1} \text { (PSK2): } \hat{m}_{n}(\alpha)=m_{n}(1) \\
& \mathrm{H}_{2} \text { (PSK4): } \hat{m}_{n}(\alpha)=m_{n}(2) \\
& \mathrm{H}_{3} \text { (PSK8): } \hat{m}_{n}(\alpha)=m_{n}(3)
\end{aligned}
$$

where $\hat{m}_{n}$ is the $n^{\text {th }}$ order moment of the estimated instantaneous phase of the received signal. Assuming a set of equal a priori probabilities $\left(P_{1}=p\left\{\mathrm{H}_{1}\right\}, P_{2}=p\left\{\mathrm{H}_{2}\right\}\right.$, and $\left.P_{3}=p\left\{\mathrm{H}_{3}\right\}\right)$, the decision rule can be formulated as follows.

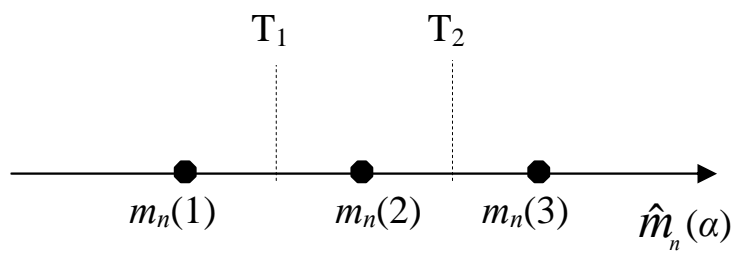




$$
\begin{array}{ll}
\hat{m}_{n}(\alpha)<T_{1} & \Rightarrow \text { decide } \mathrm{H}_{1} \\
T_{1} \leq \hat{m}_{n}(\alpha)<T_{2} & \Rightarrow \text { decide } \mathrm{H}_{2} \\
\hat{m}_{n}(\alpha) \geq T_{2} & \Rightarrow \text { decide } \mathrm{H}_{3}
\end{array}
$$

From the previous analysis, the optimum threshold, $T_{1}$, is the median value between the $n^{\text {th }}$ order moment of pure PSK2 and that of PSK4 signals; i.e.

$$
T_{1}=\frac{m_{n}(1)+m_{n}(2)}{2}
$$

Similarly, $T_{2}$ is the median value between the $n^{\text {th }}$ order moment of pure PSK4 and that of PSK8 signals; i.e.

$$
T_{2}=\frac{m_{n}(2)+m_{n}(3)}{2}
$$

In practice, the even $n^{\text {th }}$ order moment of the received signal phase can be calculated as follows

$$
\hat{m}_{n}(\alpha)=\frac{1}{N} \sum_{i=1}^{N} \varphi_{\alpha}^{n}(i) ; n \text { even }
$$

where $\varphi_{\alpha}^{n}(i)$ is the sampled $n^{\text {th }}$ order moment of the phase and $N$ is the number of samples observed in a finite interval.

Applying (10), the even $n^{\text {th }}$ order moments of the instantaneous phase of the received signal for $\alpha=1,2$, and 3 are calculated. Table 1 shows the even $n^{\text {th }}$ order moment values for $\alpha=1$ and 2 and the difference between them while Table 2 shows the even $n^{\text {th }}$ order moment values for $\alpha=2$ and 3 and the difference between them.

Table 1 . The values of the even $n^{\text {th }}$ order moments for $\alpha=1$ and 2 and the difference between them

\begin{tabular}{|c|c|c|c|c|}
\hline \multicolumn{2}{|c|}{$m_{n}(\alpha)$} & $\alpha=1$ & $\alpha=2$ & Diff. \\
\hline \multirow{4}{*}{$n$} & 2 & $0.2500 \pi^{2}$ & $0.3125 \pi^{2}$ & $0.0625 \pi^{2}$ \\
\cline { 2 - 5 } & 4 & $0.0625 \pi^{4}$ & $0.1602 \pi^{4}$ & $0.0977 \pi^{4}$ \\
\cline { 2 - 5 } & 6 & $0.0156 \pi^{6}$ & $0.0891 \pi^{6}$ & $0.0331 \pi^{6}$ \\
\cline { 2 - 5 } & 8 & $0.0039 \pi^{8}$ & $0.0501 \pi^{8}$ & $0.0462 \pi^{8}$ \\
\cline { 2 - 5 } & 16 & $0.0000 \pi^{16}$ & $0.0050 \pi^{16}$ & $0.0050 \pi^{16}$ \\
\hline
\end{tabular}


Table 2. The values of the even $n^{\text {th }}$ order moments for $\alpha=2$ and 3 and the difference between them

\begin{tabular}{|c|c|c|c|c|}
\hline \multicolumn{2}{|c|}{$m_{n}(\alpha)$} & $\alpha=2$ & $\alpha=3$ & Diff. \\
\hline \multirow{4}{*}{$n$} & 2 & $0.3125 \pi^{2}$ & $0.3281 \pi^{2}$ & $0.0156 \pi^{2}$ \\
\cline { 2 - 5 } & 4 & $0.1602 \pi^{4}$ & $0.1897 \pi^{4}$ & $0.0295 \pi^{4}$ \\
\cline { 2 - 5 } & 6 & $0.0891 \pi^{6}$ & $0.1278 \pi^{6}$ & $0.0387 \pi^{6}$ \\
\cline { 2 - 5 } & 8 & $0.0501 \pi^{8}$ & $0.0918 \pi^{8}$ & $0.0417 \pi^{8}$ \\
\cline { 2 - 5 } & 16 & $0.0050 \pi^{16}$ & $0.0297 \pi^{16}$ & $0.0247 \pi^{16}$ \\
\hline
\end{tabular}

Table 1 shows that the maximum difference between the moments of PSK2 and PSK4 occurs at the $4^{\text {th }}$ order moment. Therefore the optimum threshold, $T_{1}$, to discriminate between them is at $n=4$. Also, from table 2 it is clear that the optimum threshold, $T_{2}$, to discriminate between PSK4 and PSK8 is at $n=8$.

The proposed algorithm is executed as a computer program using the LabWindows CVI software. The functional flow chart for the proposed algorithm is shown in Fig. 2.

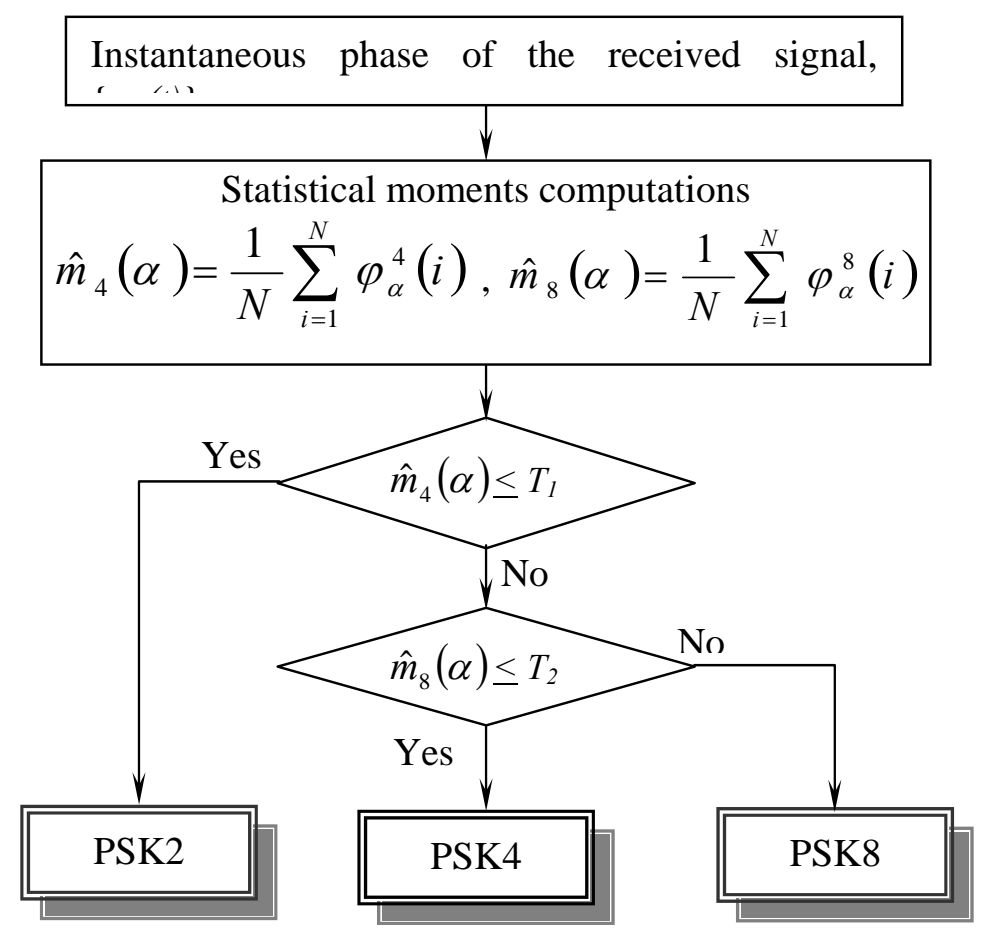

Fig. 2. Functional flowchart of the proposed MPSK classification 


\section{Performance Evaluation of MPSK Classification Algorithm}

The performance of the proposed algorithm is evaluated from 500 different realizations, each with 2048 samples, for each modulation type of interest. The MPSK signals are generated with symbol rate of $10 \mathrm{kHz}$ and a sampling rate of $100 \mathrm{kHz}$. A white Gaussian noise with power spectral density of $N_{0} / 2$ is simulated and added to the signal. The signal-to-noise ratio, SNR, is defined as the ratio of the received signal power to the noise power within the signal bandwidth.

The probabilities of correct decision for PSK2 and PSK4 classification are shown in Fig.3 and Fig.4, respectively for $n=2$, 4, and 8 .

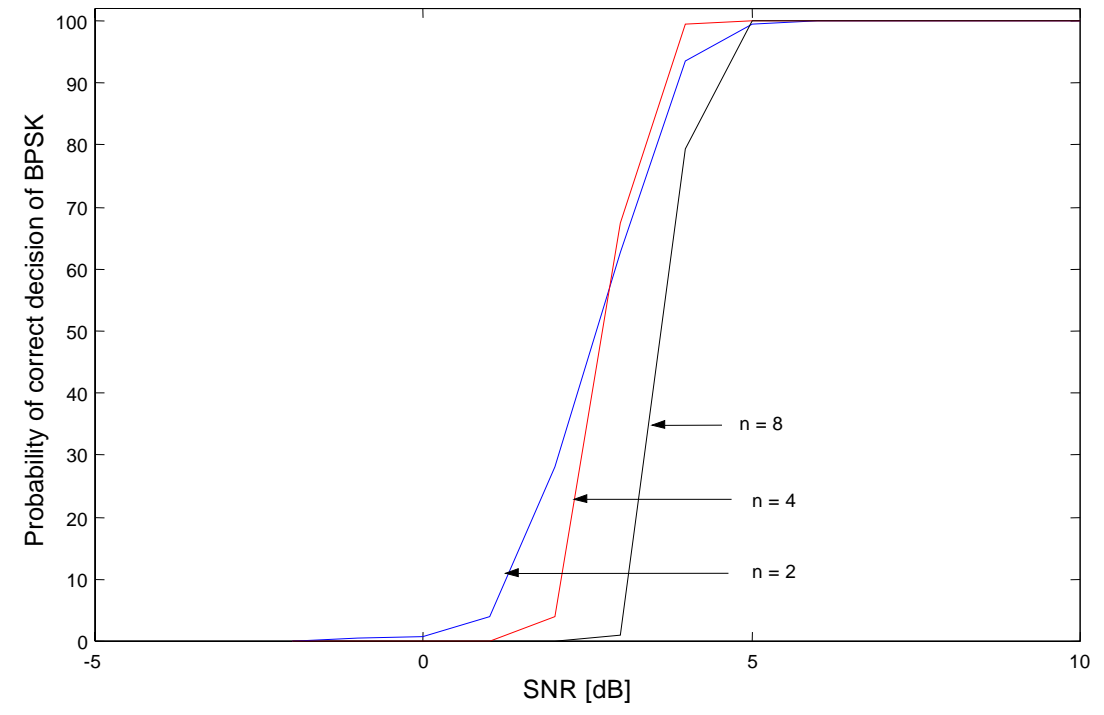

Fig.3. Probability of correct decision of PSK2

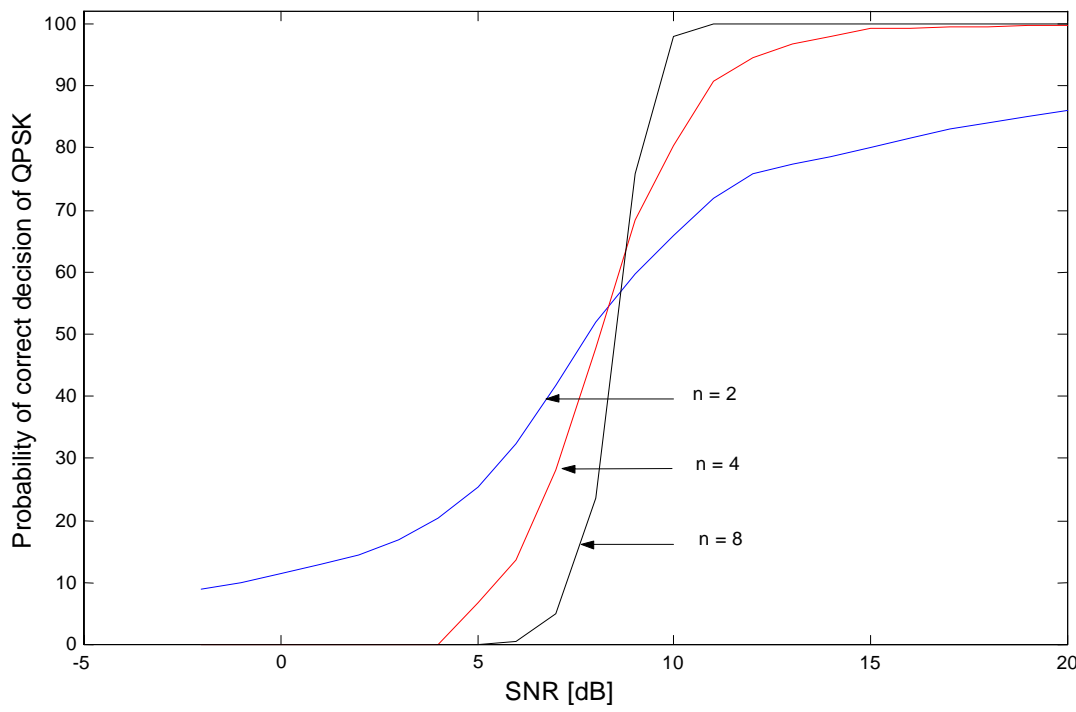

Fig.4. Probability of correct decision of PSK4 
These figures show that the best choice of $n$ for discrimination between PSK2 and PSK4 is $n$ $=4$ and for discrimination between PSK4 and PSK8 is $n=8$ which agrees with the theoretical results. All the results associated with the evaluation of the proposed method for MPSK classification are presented in tables 3and 4 at SNR of 15 and $10 \mathrm{~dB}$ respectively.

Table 3. Confusion matrix for the MPSK classifier at SNR $=15 \mathrm{~dB}$

\begin{tabular}{|c|c|c|c|c|}
\hline \multirow{2}{*}{\multicolumn{2}{|c|}{}} & \multicolumn{3}{|c|}{ Deduced Modulation Type } \\
\cline { 3 - 5 } & PSK2 & PSK4 & PSK 8 \\
\hline \multirow{3}{*}{$\begin{array}{c}\text { Simulated } \\
\text { Modulation } \\
\text { Type }\end{array}$} & PSK2 & $100 \%$ & $0.00 \%$ & $0.00 \%$ \\
\cline { 2 - 5 } & PSK4 & $0.00 \%$ & $100 \%$ & $0.00 \%$ \\
\cline { 2 - 5 } & PSK8 & $0.00 \%$ & $0.00 \%$ & $100 \%$ \\
\hline
\end{tabular}

Table 4. Confusion matrix for the MPSK classifier at SNR $=10 \mathrm{~dB}$

\begin{tabular}{|c|c|c|c|c|}
\hline \multirow{2}{*}{\multicolumn{2}{|c|}{}} & \multicolumn{3}{|c|}{ Deduced Modulation Type } \\
\cline { 3 - 5 } \multicolumn{2}{|c|}{} & PSK2 & PSK4 & PSK8 \\
\hline \multirow{3}{*}{$\begin{array}{c}\text { Simulated } \\
\text { Modulation } \\
\text { Type }\end{array}$} & PSK2 & $100 \%$ & $0.00 \%$ & $0.00 \%$ \\
\cline { 2 - 5 } & PSK4 & $0.00 \%$ & $91.11 \%$ & $8.89 \%$ \\
\cline { 2 - 5 } & PSK8 & $0.00 \%$ & $0.10 \%$ & $99.90 \%$ \\
\hline
\end{tabular}

From the results shown in tables 3 and 4, it is clear that all MPSK types of interest have been correctly classified with a success rate $\geq 91 \%$. The proposed algorithm is tested at lower value of SNR (9 dB) as shown in Table 5. The results show that at lower SNR values the proposed algorithm fails to classify PSK4 signal with an acceptable success rate but gives a high success rate for PSK2 and PSK8 as shown in Table 5. Table 6 shows the overall success rates for the proposed algorithm at different values of SNR. The overall success rate is calculated as the average of the success rates of the modulation types of interest. It is shown that for $\mathrm{SNR} \leq 8 \mathrm{~dB}$, the performance of the proposed algorithm begins to degrade.

Table 5. Confusion matrix for the MPSK classifier at SNR $=9 \mathrm{~dB}$

\begin{tabular}{||c|c|c|c|c||}
\hline \multirow{2}{*}{\multicolumn{2}{|c|}{}} & \multicolumn{3}{|c|}{ Deduced Modulation Type } \\
\cline { 3 - 5 } \multicolumn{2}{|c|}{} & PSK2 & PSK4 & PSK 8 \\
\hline \multirow{2}{*}{$\begin{array}{c}\text { Simulated } \\
\text { Modulation } \\
\text { Type }\end{array}$} & PSK2 & $100 \%$ & $0.00 \%$ & $0.00 \%$ \\
\cline { 2 - 5 } & PSK4 & $0.00 \%$ & $70.83 \%$ & $29.17 \%$ \\
\cline { 2 - 5 } & PSK8 & $0.00 \%$ & $0.10 \%$ & $99.90 \%$ \\
\hline
\end{tabular}


Table 6. Overall success rate at different SNR's

\begin{tabular}{||c|c||}
\hline SNR $[\mathrm{dB}]$ & $\begin{array}{c}\text { Overall Success Rate } \\
(\%)\end{array}$ \\
\hline 15 & 100 \\
\hline 10 & 97.00 \\
\hline 9 & 90.24 \\
\hline 8 & 78.63 \\
\hline 7 & 70.16 \\
\hline
\end{tabular}

\section{Conclusions}

An algorithm for automatic modulation classification of MPSK signals has been proposed. The proposed algorithm is based on evaluating of the even order moments of the instantaneous phase of the received PSK signal. The $4^{\text {th }}$ order moment is used to discriminate between PSK2 and PSK4. The $8^{\text {th }}$ order moment is used to discriminate between PSK4 and PSK8. The proposed algorithm has shown good classification results and a robust behavior against AWGN. The algorithm seems easily extendable to include higher levels (probably with some small modifications of the key feature extraction and the classification stages).

\section{REFERENCES}

[1] M. Richterova, "Signal modulation recognition based on method of artificial networks", Progress in Electromagnet. Research Symposium, Hangzhou, China, Aug. 2005.

[2] V. matic and V. Tadic, "Modulation classification using the power spectrum of detected signals”, Comm. System Network, CSN, benidorm, Spain, Sept. 2003.

[3] A. Swami and B. Sadler, "Hierarchical digital modulation classification using cumulants", IEEE Trans. on Communications, vol. 48, no. 3, March 2000, pp. 416-429.

[4] W. Wei and J. Mendel, "Maximum likelihood classification for digital amplitude-phase modulations”, IEEE Trans. on Communications, vol. 48, no. 2, Feb. 2000, pp. 189-193.

[5] Ahmed E. El-Mahdy, "Classification of MFSK signals over time varying flat correlated fading channels under Class-A impulsive noise”, IEE proc. on Communications, vol. 151, no. 6, December 2004, pp. 619-626.

[6] Y. Yang and S. S. Soliman, "An Improved Moment-Based Algorithm for Signal Classification “, Signal Processing, Vol. 43, No. 3, May 1995, pp. 231-249.

[7] Asoke K. Nandi and E.E. Azzouz, “ Algorithms for Automatic Modulation Recognition of Communication Signals”, IEEE trans. on Communications, Vol. 46, No. 4, April 1998, pp. 431-435.

[8] T. Helaly, "Interception of Satellite Communication Signals”, M.Sc. thesis, MTC, Cairo, Egypt, 2001. 